\title{
SOLID DOSAGE FORM DEVELOPMENT OF GLIBENCLAMIDE-ASPARTAME COCRYSTAL USING THE SOLVENT EVAPORATION METHOD TO INCREASE THE SOLUBILITY OF GLIBENCLAMIDE
}

\author{
ARIF BUDIMAN ${ }^{*}$, SANDRA MEGANTARA ${ }^{\mathrm{b}}$, AYU APRILIANI ${ }^{\mathrm{a}}$
}

aDepartment of Pharmaceutical and Technology Pharmacy, Faculty of Pharmacy, Universitas Padjadjaran, bDepartment of Pharmaceutical Analysis and Medicinal Chemistry, Faculty of Pharmacy, Universitas Padjadjaran

Email: arif.budiman@unpad.ac.id

Received: 23 Jan 2019, Revised and Accepted: 28 Mar 2018

\section{ABSTRACT}

Objective: The solubility of a drug in water plays an important role in the absorption of the drug after oral administration. Cocrystal is one method that improves the solubility of the active pharmaceutical ingredient (API). The aim of this study was to investigate the formation of a glibenclamide (GCM)-aspartame (APM) cocrystal using the solvent evaporation method and to evaluate its solubility and dissolution rate.

Methods: Molecular docking of the GCM-APM cocrystal was observed using an in silico method. The GCM-APM cocrystal (1:2) was prepared by using the solvent evaporation method. The cocrystal of GCM-APM was evaluated by the saturated solubility test and the dissolution rate test (USP type 2 apparatus). The solvent evaporation product of GCM-APM was characterized by Fourier transform infrared spectroscopy (FT-IR), differential scanning calorimetry (DSC), and powder X-ray diffraction (PXRD).

Results: In silico study showed that the interaction of GCM-APM has hydrogen bonding and the potential to improve the solubility of GCM. Evaluation of the cocrystal of GCM-APM showed that the solubility and dissolution rate of the cocrystal are significantly increased. Characterization of FT-IR showed that no chemical reaction occurred in the GCM-APM cocrystal. The DSC analysis showed the changes in the melting point of GCM. Measurement of PXRD showed the formation of a new solid crystal phase that is different from GCM and APM.

Conclusion: GCM-APM has hydrogen bonding can improve the solubility and dissolution rate of GCM.

Keywords: Cocrystal, Glibenclamide, Aspartame, Solvent Evaporation

(C) 2019 The Authors. Published by Innovare Academic Sciences Pvt Ltd. This is an open-access article under the CC BY license (http://creativecommons.org/licenses/by/4.0/) DOI: http://dx.doi.org/10.22159/ijap.2019v11i3.32121

\section{INTRODUCTION}

Solubility is an important parameter of the absorption process of a drug in the gastrointestinal tract that affects the drug concentration that can reach the blood circulation. This is important because the effectiveness of a drug treatment depends on the level of drug in the blood. Drugs with poor solubility in water exhibit poor bioavailability and low dissolution rates in the drug absorption [1]. Almost $70 \%$ of new active pharmaceutical ingredients (API) show poor water solubility, which can cause poor dissolution in the GI fluids. Therefore, increasing the dissolution rate of poorly watersoluble drugs is important in enhancing their bioavailability [2].

Glibenclamide (GCM) (5-chloro-N-[2-[4-(cyclohexyl carbamoylsulfamoyl) phenyl] ethyl]-2-methoxybenzamide) is a type 2 of antidiabetic drug used orally for controlling glycemia to treat noninsulin dependent [3]. In the biopharmaceutical classification system (BCS), GCM is included in class II, which means it has a high permeability and a low solubility. The properties of GCM can produce poor dissolution rate and can result in low bioavailability. Several studies have been conducted to increase the solubility of GCM such as solid dispersion [4], surface solid dispersion, nanoparticles [5], and nanoemulsion [6]. However, none of these methods stably yield a solid preparation.

One method that can increase the solubility of the API is cocrystal formation. Structurally, components of a cocrystal contain crystalline material present in a definite stoichiometric amount [7]. A cocrystal can improve solubility, dissolution rate, bioavailability, and stability because it has two or more neutral molecular constituents bonded together in the crystal lattice through hydrogen bonding [8]. Complex crystal formations were connected by a synthon that in pharmaceutical crystal engineering is called "a noncovalent interaction involving hydrogen bonds, Van der Waals, and $\pi-\pi$ electrons" [9]. For designing cocrystals, choosing a synthon in a cocrystal is important for the crystallization process. Using the in silico method for designing cocrystals can help predict the interaction of synthon between the API and the conformer [8].
Improving bioavailability is desirable for an antidiabetic drug, especially GCM. Hence there is strong scientific and clinical need to prepare novel forms of GCM possessing modified solubility and dissolution rate, which can be formulated in tablet dossage form. Accordingly, the aim of the present study was to prepare a glibenclamide cocrystal by using the solvent evaporation method with aspartame as a coformer. Previous studies have proved that glibenclamide-oxalic acid cocrystals increase GCM solubility by up to sixfold [10]. In addition, the use of aspartame (APM) as a coformer was shown to increase atorvastatin solubility at the rate of $136.77 \%$ compared to atorvastatin [11].

\section{MATERIALS AND METHODS}

Material

GCM was obtained from Indofarma (Indonesia) with a purity $>99 \%$ and APM pro analysis was obtained from Merck (Germany).

\section{Methods}

\section{In silico molecular docking}

Two-dimensional structures of GCM (Chem Spider ID: 54809) and coformers in. mol format were downloaded from www. chemspider. com. All. mol files of the molecules were converted into. pdb files by employing OpenBabelGUI 2.2.3. The files were then opened in AutoDock 4.2.3 and converted into. pdbq files by adding polar hydrogen and Kollman charges. The. pdbq files were converted into. pdbqt files by calculating their torsion angles, and were then ready to be used for docking. Docking was repeated five times for each coformer. Parameters observed were the type and energy (Ei) of interactions $[11,12]$

\section{Preparation of cocrystal using solvent evaporation method}

GCM and APM were weighed in equimolar ratios of 1:1 and 1:2. Each compound was dissolved in methanol and mixed for a few minutes. An equimolar solution was evaporated using a water bath at $40{ }^{\circ} \mathrm{C}$ for $48 \mathrm{~h}$. The obtained product was dried at room temperature overnight and further analyzed for its characterization [13]. 


\section{Determination of solubility}

Cocrystal GCM equivalent to $50 \mathrm{mg}$ was placed in an Erlenmeyer flask that contained water. Cocrystals were agitated in a mechanical shaker for 24 and $48 \mathrm{~h}$ at room temperature. The saturated solutions were filtered through a $0.45 \mu \mathrm{m}$ membrane filter, and the amount of the drug dissolved was analyzed spectrophotometrically at $266 \mathrm{~nm}$ [1].

\section{Optimization of pH of GCM dissolution medium}

Phosphate buffer media was prepared at $900 \mathrm{ml}$ with $\mathrm{pH}$ variations of 6.4, 7.6 and 8. The dissolution test was then performed on standard GCM using a dissolution tester type 2 (paddle) at $75 \mathrm{rpm}$ for $60 \mathrm{~min}$ at $37 \pm 0.2{ }^{\circ} \mathrm{C}$. At each time point $(5,10,15,30,45$ and 60 $\mathrm{min}$ ), samples of $5 \mathrm{ml}$ were taken and then $5 \mathrm{ml}$ of phosphate buffer media was re-added. The $5 \mathrm{ml}$ samples taken were then measured by UV spectrophotometry (Analytical Jena, Germany) so that the amount of GCM concentration and the optimal pH of the medium could both be determined [14].

\section{Dissolution test}

Cocrystals of GCM were measured with a dissolution test (USP type 2 apparatus). The sample of cocrystal was put into $900 \mathrm{ml}$ of a buffered phosphate solution with a $\mathrm{pH}$ of 8 and stirred at $75 \mathrm{rpm}$. The dissolution samples were filtered through a syringe filter of $0.45 \mu \mathrm{m}$ pore size and were measured periodically (at $0,10,15,30,45$, and $60 \mathrm{~min}$ ) and were analyzed spectrophotometrically at $266 \mathrm{~nm}$ [15].

\section{Characterization of cocrystal}

\section{Fourier transform infrared spectroscopy (FT-IR) analysis}

Samples were mixed with potassium bromide crystal and crushed until homogeneous, and then compressed to a pressure of 20 psi. The infrared spectrum was obtained using an infrared spectrophotometer in a range of wavenumbers (400-4000 cm-1) using FT-IR $[8,9,16]$

\section{Powder X-ray diffraction (PXRD) analysis}

Powder X-ray diffraction (Phillips PW1835® diffractometer) analysis was performed at room temperature . The condition of measurement was set using $\mathrm{Cu}$ Ka radiation $(\mathrm{l}=1.54 \AA)$, a tube stage of $40 \mathrm{kV}$, and a tube current of $40 \mathrm{~mA}$. Data were collected on the range of 2 theta of $5-40^{\circ}$ at a scan rate of $1-2 \% \mathrm{~min}[8,9,17]$.

\section{Differential scanning calorimetry (DSC) analysis}

Thermal analyses of cocrystal were performed on a DSC. A thermograph was recorded under a gas flow of $50 \mathrm{ml} / \mathrm{min}$. Samples were analyzed from 30 to $200{ }^{\circ} \mathrm{C}$ with a heating rate of $10{ }^{\circ} \mathrm{C} / \mathrm{min}$ $[17,18]$.

\section{Statistical analysis}

The data of the solubility test and dissolution rate test were presented as a mean of samples \pm standard deviation (SD) and were analyzed using the one-way analysis of variance (ANOVA) at the level of $(\mathrm{P}<0.05)$ to determine if the changes in the applied factors were statistically significant at the level of $(p<0.05)$ and nonsignificant at the level of $(p>0.05)$ ) [19].

\section{RESULTS}

Selection of coformers by an in silico method was used to predict the interaction between glibenclamide and a coformer molecularly. The results of an in silico method between GCM-APM and the prediction of interaction between GCM-APM can be seen in fig. 1 and fig. 2 respectively.

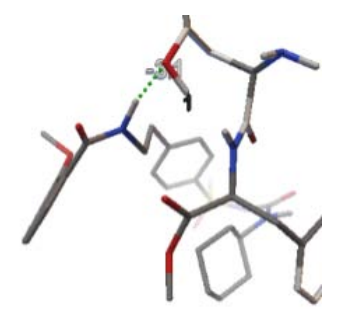

Fig. 1: In silico molecule modeling the simulation of GCM and APM

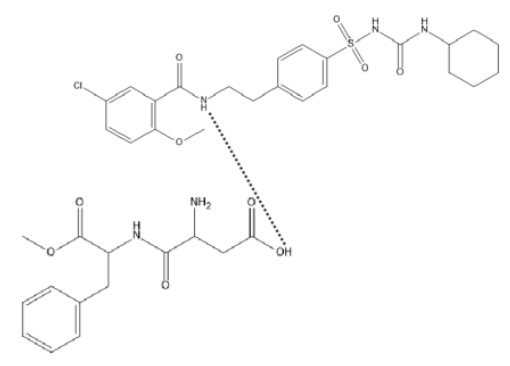

Fig. 2: The interaction between GCM and APM

The coformer used has no pharmacological activity and has an acceptor group and a hydrogen donor that is between GCM and coformer which allows the formation of a hydrogen bond [12].

The interaction of hydrogen bonds formed between GCM and APM has an effect on the hydrophilicity of GCM [20]. The hydrogen atom in the secondary amine group of GCM functions as a hydrogen bonding donor, whereas the oxygen atoms in the APM hydroxyl group function as the hydrogen bond acceptor [10].

The results of the solubility study of the GCM cocrystal can be seen in table 1.

Table 1: The result of the solubility study of the GCM cocrystal (All the values were calculated as mean \pm standard deviation) (n=3)

\begin{tabular}{|c|c|c|}
\hline \multirow[t]{2}{*}{ Sample } & \multicolumn{2}{|c|}{ Concentration $(\mu \mathrm{g} / \mathrm{ml})$} \\
\hline & $24 \mathrm{~h}$ & $48 \mathrm{~h}$ \\
\hline Pure GCM & $2.11+0.21$ & $4.40+0.12$ \\
\hline Physical mixture of GCM-APM & $13.51+0.76$ & $18.72+0.54$ \\
\hline The Solvent evaporation Product of GCM-APM (1:1) & $18.74+0.81$ & $30.48+0.78$ \\
\hline The Solvent evaporation Product of GCM-APM (1:2) & $31.71+1.15$ & $60.63+1.21$ \\
\hline
\end{tabular}

The result of the solubility test was analyzed statistically and showed that $\rho$ value was less than 0,05 ; therefore, $\mathrm{H}_{0}$ was rejected and $\mathrm{H}_{\mathrm{a}}$ was accepted, which means that there were significant differences among each treatment (pure GCM versus cocrystal of GCM).

The formation of hydrogen bonds between GCM and APM causes increased solubility of GCM. The water-soluble aspartame properties also affect the increased solubility of GCM cocrystal in water [21]. Using a coformer will decrease the barrier on the crystal so that the dissolution process of GCM becomes easier. According to the previous study when the cocrystals are ionized, the $\mathrm{pH}$ changes because the ion of the cocrystal component changes the $\mathrm{pH}$ of the environment [22].

The dissolution test was performed on the cocrystal with the best equimolar ratio based on the solubility test, i.e., GCM-APM 1:2 compared with pure GCM. Optimization of $\mathrm{pH}$ of dissolution medium was performed at $\mathrm{pH} 6.4,7.6$, and 8.0. In table 4 , the concentration of standard GCM at 60 min was 9.91, 16.43, and $45.31 \mathrm{ppm}$ for pH 6.4, 7.6, and 8.0, respectively. Based on the results, 8.0 was the optimum $\mathrm{pH}$ for the dissolution test of GCM. 


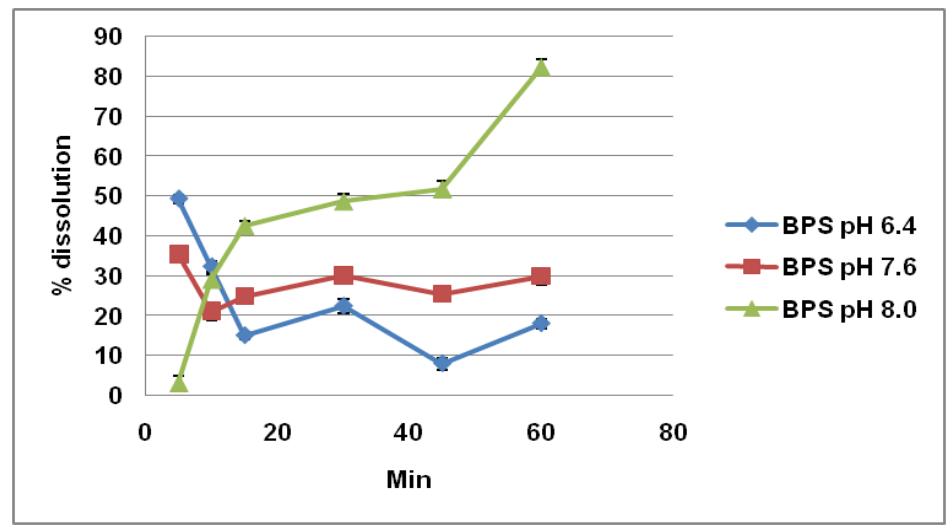

Fig. 3: Optimization of $\mathrm{pH}$ of dissolution medium (All the values were calculated as mean \pm standard deviation) ( $\mathrm{n}=3$ ), the results of the dissolution test for GCM can be seen in fig. 4

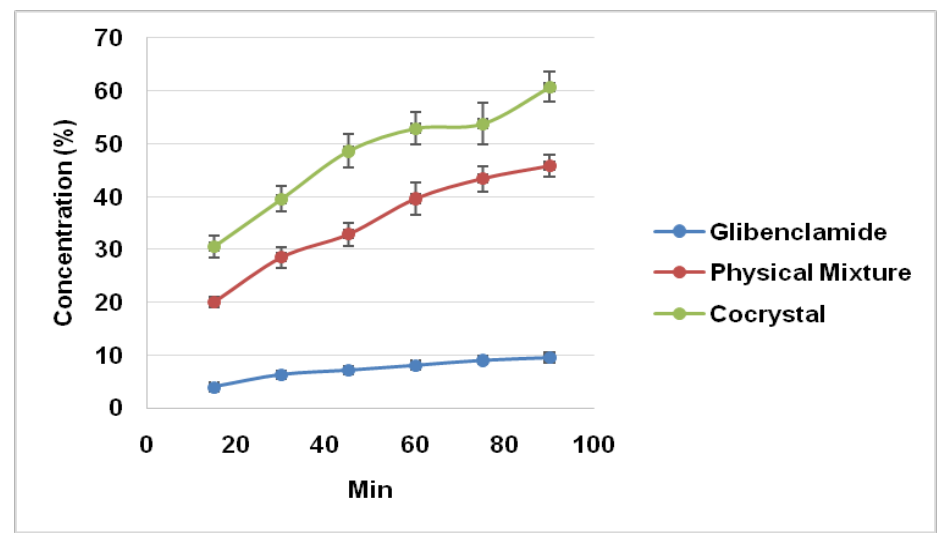

Fig. 4: The results of the dissolution test for GCM cocrystal (All the values were calculated as a mean \pm standard deviation) (n=3)

The result of the dissolution test was analyzed statistically and showed that the $\rho$ value was less than 0.05 ; therefore, $\mathrm{H}_{0}$ was rejected and $\mathrm{H}_{\mathrm{a}}$ was accepted, which means that there were significant differences among pure GCM compared to cocrystal of GCM and physical mixture.

The increase of the dissolution rate of the glibenclamide-aspartame cocrystal is caused by the hydrogen bonding interaction in the cocrystalline thus increasing the polarity of GCM In addition, the solubility of good APM in the water can affect the dissolution of GCM cocrystal [11]. Molecules in the physical mixture could become nonrandom and organized, upon heating results in lowering of melting points. It indicated that the eutectic formation was accelerated by grinding. Increased surface contact between components after grinding induces a weaker eutectic interaction which requires lower activation energy to reach the melting point [23].

The results of the FTIR spectrum from GCM, APM and cocrystal of GCM-APM can be seen in fig. 5 .

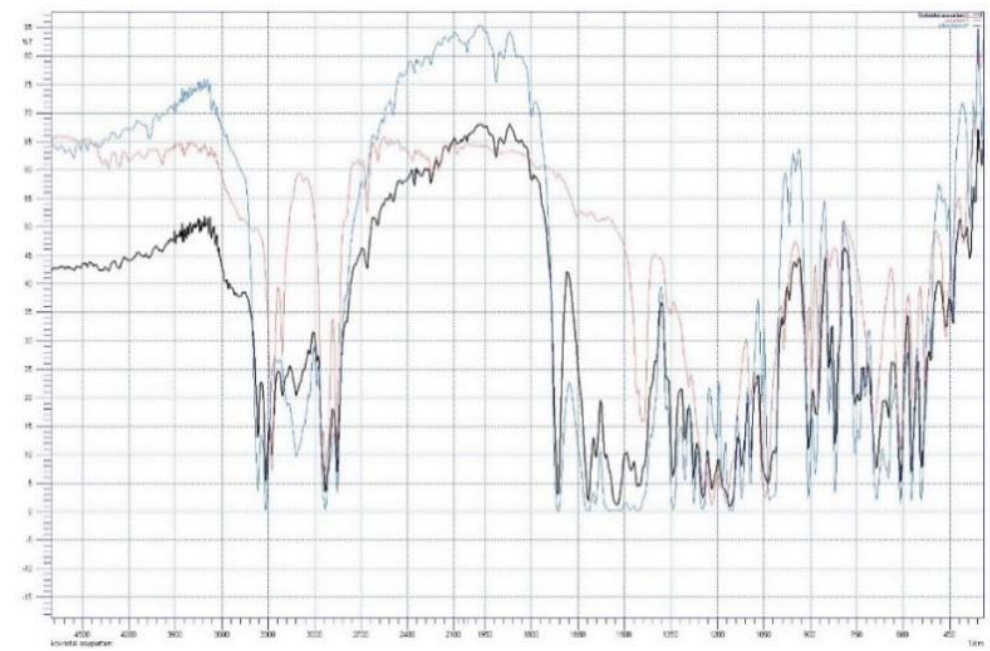

Fig. 5: The results of the FTIR spectrum of GCM (blue), APM (red), GCM-APM cocrystal (black), analysis of functional groups from the FTIR results of the GCM cocrystal can be seen in table 2 
Table 2: Analysis of functional groups from the FTIR results of the GCM cocrystal $(n=3)$

\begin{tabular}{|c|c|c|c|c|c|c|}
\hline \multirow[t]{2}{*}{ No } & \multicolumn{2}{|l|}{ GCM } & \multicolumn{2}{|l|}{ APM } & \multicolumn{2}{|c|}{ GCM-APM cocrystal } \\
\hline & Peak (cm-1) & Stretch & Peak (cm-1) & Stretch & Peak (cm-1) & Stretch \\
\hline 1 & 3313.17 & N-H Stretch & 3275.13 & N-H Stretch & 3367.71 & N-H Stretch \\
\hline 2 & 3116.97 & O-H Stretch & 2931.8 & C-H Stretch & 3205.69 & O-H Stretch \\
\hline 3 & 2931.8 & C-H Stretch & 1696.35 & $\mathrm{C}=0$ Stretch & 2854.65 & C-H Stretch \\
\hline 4 & 1712,79 & $\mathrm{C}=0$ Stretch & & & 1593.20 & $\mathrm{C}=0$ Stretch \\
\hline 5 & 1612,49 & $\mathrm{C}=\mathrm{C}$ Stretch & & & 1616.35 & $\mathrm{C}=\mathrm{C}$ Stretch \\
\hline
\end{tabular}

When the formation of functional groups was compared between GCM-APM cocrystals, GCM, and APM, there was no new peak. This indicates that there is no new functional group and it can be predicted that no chemical reaction occurred in the GCM cocrystal.

The thermogram of GCM aspartame and GCM-APM can be seen in fig. 6 .

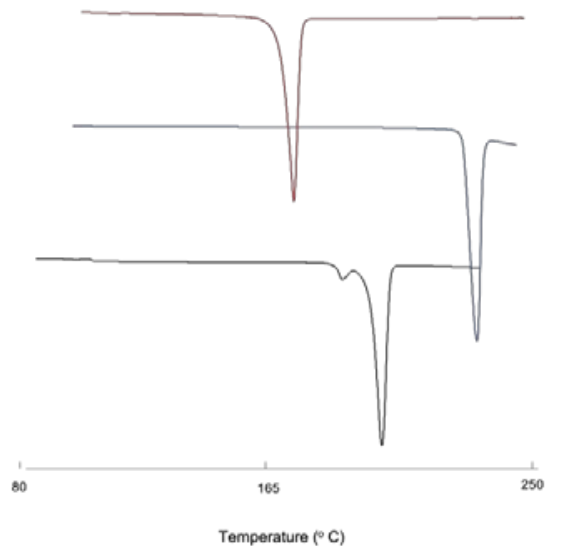

Fig. 6: The thermogram of GCM, APM, GCM-APM cocrystal

In the thermogram, there was an endothermic peak of pure GCM on $171.96{ }^{\circ} \mathrm{C}$ indicating the melting point of GCM. The endothermic peak of APM was on $240.80^{\circ} \mathrm{C}$, indicating that the APM melting point was lower than pure GCM. GCM-APM melting point also was lower than pure GCM; it was $196.65^{\circ} \mathrm{C}$. The changes in the melting point of GCM indicate the crystal changes in GCM and the possible formation of GCM cocrystal [24].

The XRD of GCM, APM and GCM-APM can be seen in fig. 7.

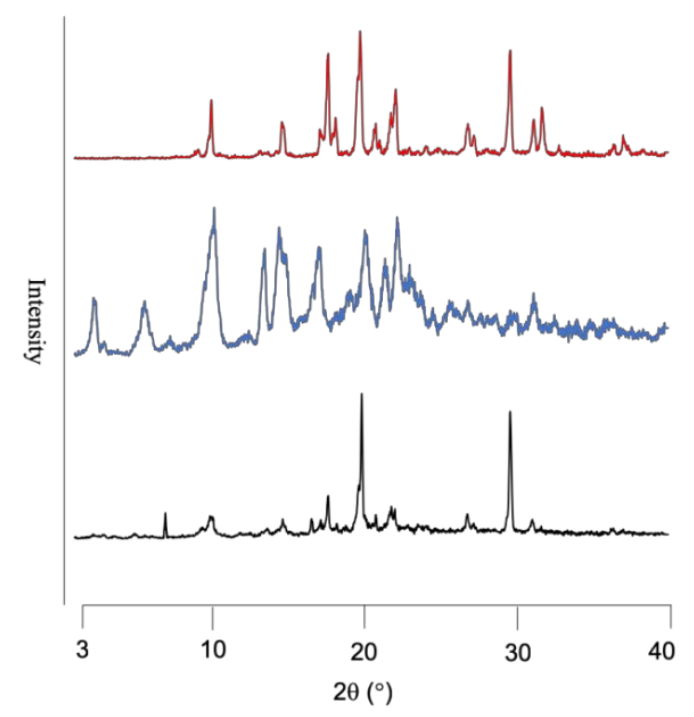

Fig. 7: The XRD of GCM (red), APM (blue) and GCM-APM (black)
The intensity diffraction pattern for the 1:2 GCM-APM cocrystal shows different diffraction pattern when compared with the intensities of GCM or APM. X-ray diffraction (XRD) was used to show differences in the shape of the cocrystal compared with pure active GCM. X-ray diffraction (XRD) is used to analyze polymorphisms and to determine changes in the properties of a cocrystal or to determine whether a new phase of the cocrystallization process has occurred. When a new phase of crystals forms or different pattern was found, the diffractogram indicates the formation of a crystal [25].

In addition, different peaks formed for pure GCM and the cocrystal $[26,27]$. It can be concluded that new peaks form and the formation of these new peaks or different diffraction pattern can predict the formation of a cocrystal $[9,10]$.

\section{CONCLUSION}

Preparation of the GCM-APM cocrystal (1:2) was conducted using the solvent evaporation method. The solubility and dissolution test of GCMAPM cocrystal (1:2) increased significantly compared to pure GCM and its physical mixture. The FT-IR, DSC, and PXRD confirmations of the cocrystal GCM-APM (1:2) indicated the formation of new solid crystalline phases that differ from pure GCM and its physical mixture.

\section{ACKNOWLEDGMENT}

The authors thank to Universitas Padjadjaran.

\section{AUTHORS CONTRIBUTIONS}

All the authors have contributed equally

\section{CONFLICTS OF INTERESTS}

All authors have none to declare

\section{REFERENCES}

1. $\mathrm{Hu}$ J, Johnston KP, Williams RO. Nanoparticle engineering processes for enhancing the dissolution rates of poorly water soluble drugs. Drug Dev Ind Pharm 2004;30:233-45.

2. Khadka P, Ro J, Kim H. Pharmaceutical particle technologies: an approach to improve drug solubility, dissolution and bioavailability. Asian J Pharm Sci 2014;9:304-16.

3. Silva Filho SF, Pereira AC, Sarraguça JMG. Synthesis of a glibenclamide cocrystal: full spectroscopic and thermal characterization. J Pharm Sci 2018;107:1597-604.

4. Tabbakhian M, Hasanzadeh F, Tavakoli N, Jamshidian Z. Dissolution enhancement of glibenclamide by solid dispersion: Solvent evaporation versus a supercritical fluid-based solventantisolvent technique. Res Pharm Sci 2014;9:337-50.

5. Dora CP, Singh SK, Kumar S, Datusalia AK, Deep A. Development and characterization of nanoparticles of glibenclamide by solvent displacement method. Acta Pol Pharm Drug Res 2010;67:283-90.

6. Bari A, Chella N, Sanka K, Shastri NR, Diwan PV. Improved antidiabetic activity of glibenclamide using oral self nano emulsifying powder. J Microencapsul 2015;32:54-60.

7. Fukte SR, Wagh MP, Rawat S. Coformer selection: an important tool in cocrystal formation. Int J Pharm Pharm Sci 2014;6:9-14.

8. Purwantoro DU, Nugrahani I, Surantaatmadja SI. Studies of preparation, characterization, and solubility of mefenamic acidnicotinamide co-crystal synthesized by using a melt crystallization method. Asian J Pharm Clin Res 2017;10:135-9.

9. Sopyan I, Fudholi A, Muchtaridi M, Sari I. Co-crystallization: a tool to enhance solubility and dissolution rate of simvastatin. J Young Pharm 2017;9:183-6. 
10. Budiman A, Nurlatifah E, Amin S. Enhancement of solubility and dissolution rate of glibenclamide by cocrystal approach with solvent drop grinding method. Int J Curr Pharm Rev Res 2016;7:248-50.

11. Gozali D, Megantara S, Levita J, Bahti HH, Soewandhi SN, Abdassah M. Virtual screening of coformers for atorvastatin cocrystallization and the characterizations of the co-crystals. Int J Pharm Sci Res 2016;7:1450-5.

12. Siswandi S, Rusdiana T, Levita J. Virtual screening of co-formers for ketoprofen co-crystallization and the molecular properties of the co-crystal. J Appl Pharm Sci 2015;5:78-82.

13. Setyawan D, Sari R, Yusuf H, Primaharinastiti R. Preparation and characterization of artesunate-nicotinamide cocrystal by solvent evaporation and slurry method. Asian J Pharm Clin Res 2014;7:62-5.

14. Gianotto EADS, Arantes RP, Lara-Filho MJ, Casimiro Filho ACS, Fregonezi-Nery MM. Dissolution test for glibenclamide tablets. Quim Nova 2007;30:1218-21.

15. Lobenberg R, Kramer J, Shah VP, Amidon GL, Dressman JB. Dissolution testing as a prognostic tool for oral drug absorption: dissolution behavior of glibenclamide. Pharm Res 2000;17:439-44.

16. Vinesha V, Sevukarajan M, Rajalakshmi R, Chowdary GT, Haritha K. Enhancement of solubility of tadalafil by cocrystal approach. Int Res J Pharm 2016;4:218-23.

17. Goyal P, Rani D, Chadha R. Crystal engineering: a remedy to tailor the biopharmaceutical aspects of glibenclamide. Cryst Growth Des 2018;18:105-18.

18. Pathak CD, Savjani KT, Gajjar AK, Savjani JK. Cocrystal formation of paracetamol with indomethacin and mefenamic acid: an efficient approach to enhance solubility. Int J Pharm Pharm Sci 2013;5:414-9.

19. Budiman A, Khoerunnisa R, Qoriah T. Wound-healing test of piper betle leaf extract and aloe vera in gel preparation. Int J Appl Pharm 2018;10:86-91.

20. Trask AV, Motherwell WDS, Jones W. Physical stability enhancement of theophylline via cocrystallization. Int J Pharm 2006;320:114-23.

21. Qiao N, Li M, Schlindwein W, Malek N, Davies A, Trappitt G. Pharmaceutical cocrystals: an overview. Int J Pharm 2011;419:1-11.

22. Thakuria R, Delori A, Jones W, Lipert MP, Roy L, Rodriguez Hornedo N. Pharmaceutical cocrystals and poorly soluble drugs. Int J Pharm 2013;453:101-25.

23. Shete A, Murthy S, Korpale S, Yadav A, Sajane S, Sakhare S, et al. Cocrystals of itraconazole with amino acids: Screening, synthesis, solid-state characterization, in vitro drug release and antifungal activity. J Drug Delivery Sci Technol 2015;28:46-55.

24. Huang X, Lai Y, Hang ZH, Zheng H, Chan CT. Dirac cones induced by accidental degeneracy in photonic crystals and zero-refractive-index materials. Nat Mater 2011;10:582-6.

25. Prohens R, Puigjaner Vallet MC. Crystal engineering studies: polymorphs and co-crystals. Handb Instrum Tech from CCiTUB; 2012. p. $45-124$.

26. Musumeci D, Hunter CA, Prohens R, Scuderi S, McCabe JF. Virtual cocrystal screening. Chem Sci 2011;2:883-90.

27. Budiman A, Megantarab S, Raraswati P, Tazyinul Qoriah A Solid dosage form development of glibenclamide with increasing the solubility and dissolution rate using cocrystallization. Int J Appl Pharm 2018;10:181-6. 